\title{
Avaliação da Efetividade dos Cursos Preparatórios para Residência Médica
}

Evaluation of the Effectiveness of Preparatory
Courses for Medical Residency Admission Tests

\author{
Isabel Cristina Gonçalves Leite ${ }^{I}$ \\ Maria Teresa Bustamante Teixeira ${ }^{I}$ \\ Hugo Silva Neves ${ }^{l}$ \\ Leonardo de Resende Sousa Oliveiral \\ Luiz Alberto Otoni Garcial \\ Pedro Henrique Martins da Cunha
}

\section{PALAVRAS-CHAVE: \\ - Internato e residência; \\ - Ensino; \\ - Aprendizagem.}

\section{KEY WORDS:}

- Internship and residency;

- Teaching;

- Learning.
Reencaminhado em: 21/01/2008

Aprovado em: 16/01/2008

\section{RESUMO}

No Brasil, há um déficit de vagas para Residência Médica (RM) e maior concorrência em determinadas especialidades ou regiões, propiciando o crescimento dos Cursos Preparatórios (CPs) para provas de RM. Os acadêmicos, cada vez mais, deixam atividades curriculares em segundo plano e se matriculam nesses cursinhos. Mediante um questionário dirigido aos candidatos do concurso de RM do Hospital Universitário da Universidade Federal de Juiz de Fora (HU-UFJF), realizou-se um estudo para avaliar a efetividade da preparação de alunos em CPs. A adesão foi de 74,7\% dos 368 concorrentes, sendo que a maioria freqüentou CPs (72\%). Não houve diferença significativa de aprovação entre os candidatos que se prepararam em CPs e os que não os freqüentaram. Já a média das notas dos candidatos que freqüentaram CPs foi 4,07 pontos maior (66,89 contra 62,82, $p<0,05)$. Conclui-se que, no modelo tradicional de avaliação, cuja prova teórica corresponde a $90 \%$ da nota final, a preparação dos alunos que freqüentaram CPs é mais eficaz, o que enaltece a memorização em detrimento de habilidades médicas. Nesse contexto, pode-se considerar o modelo 50/50, em que a prova prática corresponde a 50\% da nota final, uma proposta adequada.

\section{ABSTRACT}

In Brazil there are not enough vacancies for Medical Residency (MR) and a very high competition for vacancies in certain specialties and regions. This favors the establishment of Preparatory Courses (PCs) for MR tests. Medical students have been increasingly neglecting their curricular activities for enrolling in these Preparatory Courses. For evaluating the effectiveness of these courses, a questionnaire was applied to the candidates for MR at the University Hospital of Federal University of Juiz de Fora. $74.7 \%$ from the 368 applicants agreed to participate in the study, most of which (72\%) were frequenting PCs. There was no significant difference in the rate of approval among the candidates who had prepared themselves in PCs and those who did not. On the other hand, the average grade of the candidates that took PCs was 4.07 points higher (66.89 versus $62.82, p<0.05$ ). It is concluded that in the traditional evaluation model, whose theoretical test corresponds to $90 \%$ of the final grade, the preparation of the students who took PCs is more effective, valuing memorization above medical abilities. Thus, the 50/50 model, in which the practical test corresponds to $50 \%$ of the final grade, can be considered an adequate proposal. 


\section{INTRODUÇÃO}

O déficit de vagas para Residência Médica (RM) no Brasil e a maior concorrência em determinadas especialidades ou regiões do País têm sido responsáveis pelo crescimento da oferta de um tipo de serviço que preocupa as entidades médicas e especialistas: os Cursos Preparatórios (CPs) para provas de $\mathrm{RM}$, que funcionam nos moldes dos cursinhos pré-vestibulares, têm fins lucrativos e oferecem aulas intensivas.

A concepção do meio acadêmico em relação aos CPs é controversa e motivo de intenso debate. Essas empresas consideram-se veículo de transmissão de conhecimento de forma otimizada e estariam aptas a conciliar aprendizado com melhores resultados nos concursos de RM. Para isso, afirmam dispor de material didático atualizado, listas de exercício, simulados e cursos extras para preparação do aluno para provas escritas, prática e entrevista. Em acréscimo, cercam-se de infra-estrutura que inclui auditórios e transmissão de aulas via satélite, sendo que alguns ainda oferecem aulas via internet ${ }^{1}$. Revelam, pois, intenção de complementar e sistematizar o estudo da Medicina, visando preparar o estudante para os exames de $\mathrm{RM}^{2}$. As residências mais concorridas e que costumam ter mais alunos matriculados nesses cursinhos são: Dermatologia, Ortopedia, Oftalmologia e Clínica Médica. O módulo completo do curso dura, em média, um ano e custa entre $\mathrm{R} \$ 350$ e R $\$ 500$ por mês ${ }^{3}$.

Entre a maioria dos docentes, há a preocupação com a constituição de um círculo vicioso, que envolve os seguintes aspectos: falhas na formação médico-acadêmica, um método de avaliação para concursos de RM eminentemente teórico e a busca da suplementação em CPs. Trata-se de uma tríade que acarreta, no final, maior deterioração na formação médica, principalmente no que diz respeito à formação de habilidades e à incorporação da ética médica, já que o aluno é desestimulado a se dedicar ao internato ${ }^{4}$.

Para a grande maioria dos alunos, no entanto, os cursinhos tornaram-se parte da formação acadêmica. Na Universidade Federal de Juiz de Fora, cerca de três em cada quatro alunos de Medicina do sétimo, oitavo e nono períodos declaram intenção de se matricular nos CPs, tendo como principal objetivo a atualização e/ou revisão do conteúdo da prova. Entre os que não pretendem se matricular, a principal razão é a falta de condições financeiras ${ }^{5}$.

A prova prática como estratégia de seleção de candidatos aos programas de RM surgiu como proposta em 2004, após debates na Associação Brasileira de Educação Médica (Abem) e na Comissão Nacional de Residência Médica (CNRM). A incorporação deste tipo de avaliação nos processos seletivos tem por fundamento o reconhecimento do efeito deletério da seleção, da forma como é feita, sobre o estudante de Medicina e conseqüentemente sobre a graduação. $\mathrm{O}$ modelo tradicional, ainda adotado pela maioria das instituições brasileiras, assim como no processo seletivo do HU-UFJF, é composto por uma avaliação teórica que corresponde a $90 \%$ da nota final, sendo os $10 \%$ restantes obtidos por meio de entrevista, avaliação de currículo e/ou prova prática. Trata-se do modelo 90/10.

No modelo proposto pela Abem e pela CNRM, a prova teórica corresponde a $50 \%$ da nota final. Os 50\% restantes podem ser alcançados exclusivamente por meio de prova prática ou poderiam ser divididos em $40 \%$ para avaliação prática e $10 \%$ para entrevista e análise de currículo. Fica caracterizado, assim, o modelo $50 / 50^{6}$.

Algumas escolas médicas brasileiras, sendo 93\% delas públicas, já realizaram provas práticas, e as experiências foram discutidas em 2005 na Oficina Nacional de Avaliação da Prova Prática. Trata-se de uma questão controversa. A recomendação dessa Oficina é aguardar de três a cinco anos para reavaliar a definição desse tipo de prova, sua execução e seu impacto no ingresso à Residência Médica. O maior consenso foi quanto à não obrigatoriedade da instituição da prova prática nos concursos brasileiros, devendo permanecer o caráter facultativo nesse período ${ }^{7}$.

Embora a opinião da maioria dos professores sobre essas empresas seja desfavorável, os alunos, preocupados em passar nas provas, deixam cada vez mais as atividades curriculares em segundo plano e se matriculam nesses cursinhos. Essa adesão, cada vez mais freqüente, merece atenção e desperta questionamento sobre a efetividade da preparação de alunos em Cursos Preparatórios.

O objetivo do presente estudo é verificar se há diferença de desempenho entre os alunos que cursaram ou não CPs na preparação para o concurso de Residência Médica para preenchimento de vagas no HU-UFJF em 2007. Este elemento constitui, assim, um indicador de efetividade dos cursinhos na preparação para a prova de Residência Médica.

\section{A RESIDÊNCIA MÉDICA: DA HISTÓRIA À ATUALIDADE}

A Residência Médica nasceu em 1889, no Departamento de Cirurgia do Hospital John's Hopkins, nos Estados Unidos, para atender ao desenvolvimento das especialidades médicas. No Brasil, foi implantada na década de 1940 no Hospital das Clínicas da Faculdade de Medicina da Universidade de São Paulo, mas foi somente na década de 1970 que ocorreu sua verdadeira expansão ${ }^{8}$. A regulamentação do processo de formação dos médicos passou a ser executada, em 1977, pela Comissão Nacional de Residência Médica (CNRM), órgão criado pelo Decreto $n^{\circ} 80.281^{9}$.

A RM, considerada o padrão ouro da especialização médica, é definida como modalidade de ensino de pós-graduação lato sensu, na forma de curso de especialização em instituições de saúde, sob a orientação de profissionais médicos de eleva- 
da qualificação ética e profissional. O Programa de RM, cumprido integralmente dentro de determinada especialidade, confere ao médico residente o título de especialista.

Abrangendo a definição de especialização, a RM pode ser considerada fundamental para a consolidação do conhecimento médico, geral ou específico. Desta forma, a possibilidade de sua oferta deve atender dois quesitos: a demanda decorrente do número de médicos graduados e as necessidades de serviços médicos. O segundo quesito tem relação com definições do sistema de saúde, bem como com demandas da sociedade, traduzidas na procura pela assistência à saúde ${ }^{10}$.

No Brasil, 360 instituições oferecem mais de 2.600 Programas de Residência Médica, nas 54 especialidades reconhecidas no País, num total de aproximadamente 27 mil vagas, com duração de no mínimo dois anos e no máximo seis anos ${ }^{11}$. Entretanto, a maioria $(68,8 \%)$ das vagas, instituições e programas está nas capitais dos Estados, com exceção do Estado de São Paulo, onde a distribuição é mais uniforme ${ }^{12}$.

Conforme estudo conduzido pela Abem, as vagas de RM existentes no Brasil não acompanharam o crescimento de formandos e persistem concentradas em determinadas regiões e grandes centros. Entre 2002 e 2005, nove programas tiveram sua duração aumentada em um ano e apenas 15 foram criados. Hoje, o número de vagas da RM atende pouco mais de $70 \%$ dos cerca de 12 mil alunos que se formam anualmente no País e estão assim distribuídas, por região: $1,9 \%$ no Norte; $7,1 \%$ no Centro-Oeste; $11,6 \%$ no Nordeste, $15,9 \%$ no Sul; e $63,5 \%$ no Sudeste ${ }^{13}$.

\section{MATERIAL E MÉTODO}

Trata-se de um estudo transversal baseado no desempenho dos candidatos do concurso de Residência Médica 2007 do Hospital Universitário da Universidade Federal de Juiz de Fora (HU-UFJF), realizado em dezembro de 2006. A primeira página da avaliação escrita da primeira etapa do processo seletivo apresentou a cada candidato um questionário de seis perguntas objetivas (Anexo). Por meio de algumas delas, pôde-se extrair as informações específicas para a execução do presente estudo.

As perguntas foram respondidas individual e voluntariamente por $74,7 \%$ dos 368 concorrentes às vagas oferecidas nas Residências Médicas de entrada direta, sendo essas as RM que não exigem outras especialidades como pré-requisito. Constituiu-se, portanto, uma amostra de 275 pessoas que concorreram às seguintes especialidades médicas: Anestesiologia, Clínica Médica, Cirurgia Geral, Dermatologia, Ginecologia e Obstetrícia, Neurologia, Medicina de Família e Comunidade, Patologia, Pediatria e Psiquiatria. Haja vista a pequena amostra, Residências que exigem outras especialidades como pré-requisito para seu ingresso foram desconsideradas: Cardiologia, Gastroenterologia,
Hematologia, Nefrologia, Pneumologia, Cirurgia Torácica, Cirurgia Plástica, Urologia, Endoscopia Digestiva e Hepatologia.

O resultado do concurso divulgou as notas dos candidatos, assim como a colocação deles nesse processo seletivo. Todos os dados foram armazenados e analisados pelo programa EPI-Info 3.3.2, sendo que a análise descritiva utilizou a comparação de proporções e médias por meio dos testes $X^{2}$ e $t$, respectivamente, com $\mathrm{p}$-valor significativo de $5 \%$.

A efetividade na preparação individual para o concurso pôde ser evidenciada pelo sucesso do candidato, que é passível de ser medido tanto pela aprovação, quanto pela obtenção de boas notas. Entretanto, devido à desigual distribuição dos candidatos, da concorrência e da preparação nas diversas especialidades, os critérios nota do candidato e aprovação serão analisados separadamente, de modo a minimizar possíveis vieses.

No primeiro, utiliza-se a nota final obtida na avaliação escrita nas diversas especialidades. No que diz respeito à aprovação em determinada especialidade, foram considerados aprovados os candidatos que responderam o questionário proposto e que foram classificados entre os primeiros colocados até a posição $n$, sendo $n$ igual ao número de vagas de cada RM discriminadas no edital do concurso. No caso de empate entre o último aprovado e outro(s) candidato(s), esse(s) também foi(foram) considerado(s) aprovado(s).

Partindo-se do pressuposto de que se objetiva obter estatísticas significativamente válidas e avaliar a efetividade na preparação do aluno pelos CPs, considerou-se inadequado utilizar dados referentes à segunda etapa, os quais têm $10 \%$ de peso de análise curricular. Assim, esta fase do concurso foi desconsiderada, sendo o único critério para a aprovação a prova objetiva da primeira etapa, já que esta é universal nesse concurso e avalia especificamente o conhecimento do concorrente.

Por fim, estratificaram-se as RM em Grupos I e II, sendo o primeiro composto pelas mais concorridas (consideradas as de relação candidato/vaga maior que 6), e o segundo, pelas Residências menos concorridas (relação candidato/vaga menor ou igual a 6).

\section{RESULTADOS E DISCUSSÃO}

A essência da formação médico-acadêmica deve se basear na excelência do exercício da medicina, na geração e disseminação do conhecimento científico e de práticas de intervenção que expressem efetivo compromisso com a melhoria da saúde e com os direitos das pessoas ${ }^{14}$. Idealmente, a seleção de candidatos em concursos para RM deve corroborar esse princípio acadêmico, propiciando maior dedicação dos alunos às atividades curriculares e maior autonomia do corpo docente na escolha da metodologia de ensino. No entanto, no Brasil, os processos de avaliação para o ingresso em programas de RM são geralmente conduzi- 
dos de modo a privilegiar a memorização de conceitos, sendo as provas de múltipla escolha o principal recurso utilizado.

A prova de RM tem grande influência no desempenho do aluno de Medicina durante a faculdade, sendo que muitos, erroneamente, acreditam que esta é a síntese das dificuldades que terão após a formação médica. Trata-se de uma exigência essencialmente teórica que, além de desestimular o aluno no desenvolvimento da relação médico-paciente, no contato com doenças prevalentes na comunidade e no desenvolvimento de habilidades práticas nos hospitais universitários ou faculdades, ainda colabora para a disseminação dos Cursos Preparatórios para RM"15.

A partir de dados do processo seletivo 2007 do concurso de Residência Médica do HU-UFJF, observou-se a grande propagação dessas empresas no meio acadêmico. A maioria dos alunos (72\%) relata ter cursado CPs, sendo o tempo médio de freqüência igual a 11,2 meses. Confirma-se a proporção constatada em estudo prévio realizado nessa mesma universidade, em que três em cada quatro alunos dos sétimo, oitavo e nono períodos da UFJF expressaram interesse de se matricular em CPs. Nesse mesmo trabalho, constatou-se que o último ano da faculdade é o período em que a maioria dos alunos pretende se matricular ${ }^{5}$. Em conseqüência, fica justificado por que o internato é a etapa da formação mais intensamente prejudicada pela crescente procura pelos cursinhos ${ }^{16}$ (Tabela 1).

Em paralelo, discute-se no Congresso Nacional e nos Conselhos Médicos a criação do Exame de Proficiência em Medicina, nos moldes do Exame de Ordem brasileiro e do United States Medical Licensing Examination (USMLE), exame de permissão para exercício médico nos Estados Unidos. Trata-se de uma proposta polêmica e que provavelmente estimularia maior crescimento
TABELA 1

Freqüiência da preparação em Cursos Preparatórios (CPs)

\begin{tabular}{lccc}
\hline & $\begin{array}{c}\text { Freqüentou algum CP } \\
\text { para prova de RM? }\end{array}$ & Freqüência & Percentagem \\
\hline Sim & & 198 & 72 \\
Não & 77 & 28 \\
Total & & 275 & 100,0 \\
\hline
\end{tabular}

dessas empresas e uma adesão mais precoce de alunos a esses cursinhos, o que afastaria ainda mais o estudante da formação curricular, prejudicando a formação médico-acadêmica ${ }^{2}$.

Na distribuição de alunos segundo a especialidade desejada, verifica-se maior procura por Clínica Médica, Cirurgia Geral e Dermatologia, Residências almejadas por $62,9 \%$ dos participantes. Uma vez que as duas primeiras são consideradas as principais RM pré-requisitadas para o ingresso em outras especialidades, justifica-se a grande procura por elas. Dermatologia, embora não siga esse critério, é a RM de maior disputa, sendo a concorrência de 36 candidatos para cada vaga.

Ao se relacionar o nível de dificuldade para aprovação nas diferentes especialidades com a prevalência de procura pelos $\mathrm{CPs}$, constata-se, percentualmente, que a maior parte da clientela dessas empresas é constituída por alunos ou médicos que concorrem por vagas em RM mais disputadas. Recorreram aos CPs com a finalidade de se preparar para o processo seletivo $74,5 \%$ dos candidatos para especialidades mais concorridas (Grupo I), e 58,1\% daqueles pertencentes ao Grupo II, sendo que a Pediatria é uma exceção (Tabela 2).

Como já explicitado em "Material e Método", a avaliação da efetividade dos CPs será mensurada pela aprovação do

TABELA 2

Distribuição dos candidatos no concurso de RM, levando em consideração freqüência, relação candidato/vaga e prevalência de candidatos que se prepararam em CPs em cada especialidade

\begin{tabular}{|c|c|c|c|c|c|}
\hline & Especialidade & Freqüência & Percentagem & Candidato/Vaga & $\begin{array}{l}\text { Candidatos que } \\
\text { freqüentaram CPs }\end{array}$ \\
\hline$\overline{\mathrm{G}}$ & Anestesiologia & 22 & 8,0 & 13 & 81,8 \\
\hline $\mathrm{R}$ & Cirurgia Geral & 49 & 17,7 & 17,5 & 77,6 \\
\hline $\mathrm{U}$ & Clínica Médica & 76 & 27,6 & 15,5 & 76,3 \\
\hline $\mathrm{P}$ & Dermatologia & 48 & 17,5 & 36 & 75 \\
\hline $\mathrm{O}$ & Neurologia & 22 & 8,0 & 14 & 63,6 \\
\hline I & Psiquiatria & 15 & 5,5 & 11 & 60 \\
\hline \multicolumn{2}{|c|}{ Média Grupo I } & 232 & 84,3 & 17,8 & 74,5 \\
\hline G & Ginecologia e Obstetrícia & 14 & 5,2 & 4,5 & 42,9 \\
\hline $\mathrm{R}$ & Medicina da Família e & 11 & 4,0 & & \\
\hline $\mathrm{U}$ & Comunidade & & & 2 & 45,5 \\
\hline $\mathrm{P}$ & Patologia & 4 & 1,5 & 6 & 50 \\
\hline $\mathrm{O}$ & Pediatria & 14 & 5,1 & 3,8 & 85,7 \\
\hline \multicolumn{6}{|l|}{ II } \\
\hline \multicolumn{2}{|c|}{ Média Grupo II } & 43 & 15,7 & 4 & 58,1 \\
\hline \multicolumn{2}{|c|}{ Total e média geral } & 275 & 100,0 & 10,51 & 72 \\
\hline
\end{tabular}


TABELA 3

Freqüiência e prevalência de aprovação de alunos, total e por especialidade

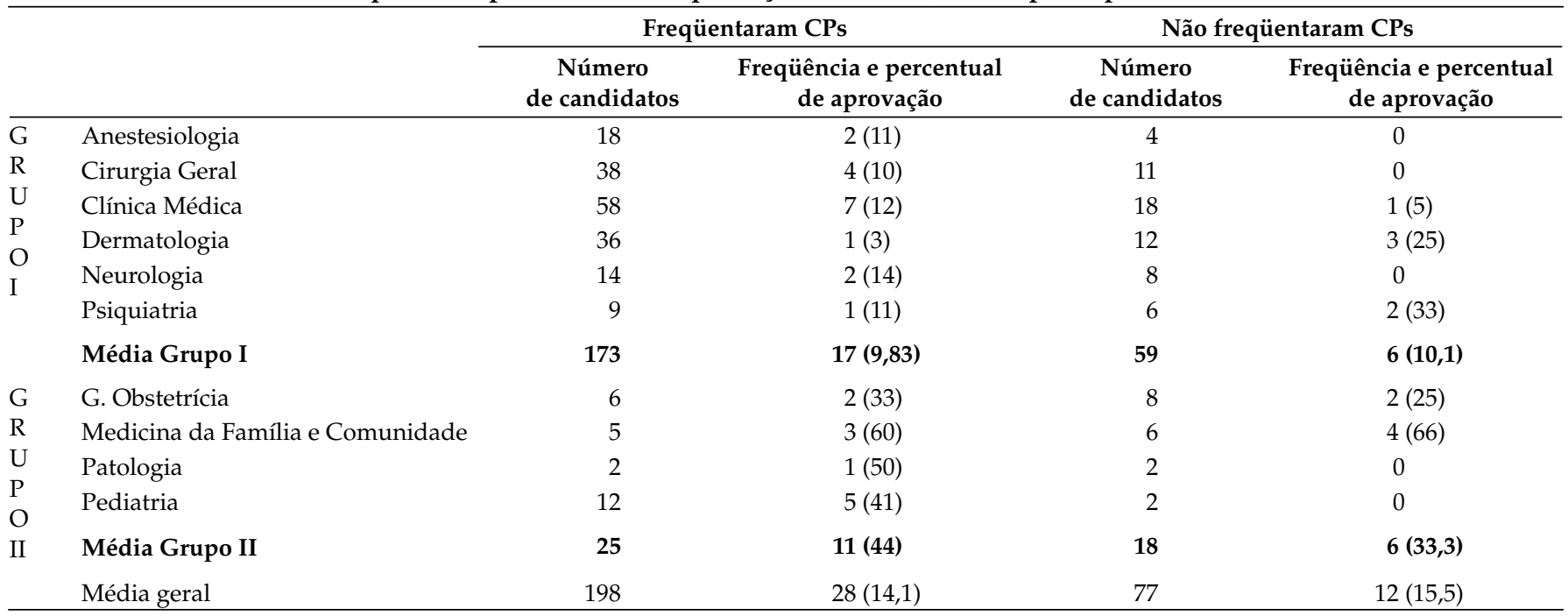

candidato e pela nota obtida na prova, de forma independente, objetivando-se minimizar possíveis vieses.

Primeiramente, analisemos os resultados relativos à aprovação. Na análise da média geral, não foi observada diferença significativa de aprovação no processo seletivo entre os candidatos que se preparam em CPs e os que não freqüentaram tais cursinhos, bem como na estratificação em Grupos I e Grupo II. ( $p$ valores $=0,94$ e 0,48 , respectivamente).

Entretanto, observa-se que, em sete das dez RMs analisadas, a aprovação é, percentualmente, maior no grupo das pessoas que freqüentaram os CPs para Residência Médica. Trata-se de uma maioria expressiva, embora seja essencial ressaltar que não há diferença significantemente estatística para o estudo da efetividade dos CPs para cada especialidade, individualmente.

No que diz respeito ao desempenho do aluno baseado na nota, fica evidenciado que a média das notas dos candidatos que freqüentaram os CPs é 4,07 pontos maior. Nesse critério, portanto, considera-se eficaz a preparação de alunos em Cursos Preparatórios nas especialidades dos Grupos I e II (Tabela 4).

TABELA 4

Média das notas dos alunos nas diversas especialidades e notas mínimas necessárias à aprovação

\begin{tabular}{|c|c|c|c|c|c|}
\hline & RM escolhida & $\begin{array}{l}\text { Média de notas dos alunos } \\
\text { que freqüentaram CPs }\end{array}$ & $\begin{array}{l}\text { Média de notas dos alunos } \\
\text { que não freqüentaram CPs }\end{array}$ & $p$ valores & $\begin{array}{c}\text { Nota mínima para } \\
\text { aprovação }\end{array}$ \\
\hline \multirow{7}{*}{$\begin{array}{l}\mathrm{G} \\
\mathrm{R} \\
\mathrm{U} \\
\mathrm{P} \\
\mathrm{O} \\
\mathrm{I}\end{array}$} & Anestesiologia & 67,55 & 58,00 & 0,001 & 74 \\
\hline & Cirurgia Geral & 68,02 & 65,72 & 0,02 & 79 \\
\hline & Clínica Médica & 66,44 & 64,22 & 0,003 & 77 \\
\hline & Dermatologia & 69,30 & 71,66 & 0,04 & 78 \\
\hline & Neurologia & 71,35 & 62,62 & 0,001 & 81 \\
\hline & Psiquiatria & 61,66 & 61,83 & 0,9 & 68 \\
\hline & Média Grupo I & 67,38 & 64 & 0,001 & 76,15 \\
\hline \multirow{4}{*}{$\begin{array}{l}\mathrm{G} \\
\mathrm{R} \\
\mathrm{U} \\
\mathrm{P} \\
\mathrm{O} \\
\mathrm{II}\end{array}$} & G. Obstetrícia & 57,5 & 55,37 & 0,19 & 63 \\
\hline & Medicina da Família e Comunidade & 58,4 & 59,83 & 0,42 & 57 \\
\hline & Patologia & 63,5 & 55,5 & 0,03 & 67 \\
\hline & Pediatria & 64,75 & 41 & 0,001 & 68 \\
\hline & Média Grupo II & 61,03 & 52,92 & 0,001 & 63,75 \\
\hline & Média geral & 66,89 & 62,82 & 0,0002 & 69,95 \\
\hline
\end{tabular}


Em cursos como Anestesiologia, Cirurgia Geral, Clínica Médica, Neurologia, Patologia e Pediatria, houve média significativamente maior por parte dos alunos que freqüentaram CPs. Por outro lado, Dermatologia é o único curso cujos alunos que não freqüentaram Cursos Preparatórios tiveram um desempenho médio significativamente melhor $(\mathrm{p}<0,05)$. Nas outras especialidades, não houve diferença média significativa ( $p>0,05)$.

A apresentação das notas em intervalos (Tabela 5) permite uma visualização clara da diferença de desempenho dos candidatos. Devem ser ressaltados os intervalos de notas 71-80 e 51-60, os únicos que apresentaram diferenças de notas estatisticamente significativas $(\mathrm{p}<0,05)$ e, portanto, estratégicos na análise diferencial de desempenho dos concorrentes.

O primeiro intervalo é composto por pessoas que estão bem colocadas no concurso e que têm chances potenciais de serem aprovadas nesse processo seletivo (de acordo com as notas mínimas para aprovação apresentadas na Tabela 5). Indubitavelmente, esse fato depende da especialidade escolhida no momento de suas inscrições no concurso. A maioria dos candidatos que tiveram notas entre 51 e 60, por outro lado, foi reprovada.

TABELA 5

Distribuição dos alunos que freqüentaram ou não CPs segundo as notas

\begin{tabular}{crrrrl}
\hline Nota & \multicolumn{2}{c}{ Freqüentaram CPs } & Não freqüentaram CPs & p valores \\
\hline$>80$ & 3 & $1,5 \%$ & 1 & $1,3 \%$ & 0,67 \\
$71-80$ & 68 & $34,4 \%$ & 17 & $22 \%$ & 0,048 \\
$61-70$ & 88 & $44,4 \%$ & 31 & $40,3 \%$ & 0,53 \\
$51-60$ & 32 & $16,2 \%$ & 21 & $27,4 \%$ & 0,03 \\
$<51$ & 7 & $3,5 \%$ & 7 & $9 \%$ & 0,11 \\
\hline Total & 198 & $100,0 \%$ & 77 & $100 \%$ & \\
\hline
\end{tabular}

Ao se analisar o rendimento dos candidatos nesses dois intervalos, nota-se que há maior número de concorrentes que freqüentaram CPs na faixa de notas entre 71 e 80, o que, por conseqüência, revela melhor desempenho desse grupo. Por outro lado, o intervalo composto por notas de 51 a 60 é composto majoritariamente por pessoas que não freqüentaram os cursinhos, corroborando essa diferença.

A constatação de que a média das notas dos alunos que freqüentaram Cursos Preparatórios é maior (4,07 pontos) e de que não há diferença significativa de aprovação de candidatos, sejam eles freqüentadores ou não desses cursinhos, é curiosa e exige discussão específica.

A parcela de alunos que freqüentam CPs procurou, em sua maioria (87,3\%), por especialidades mais concorridas (Tabela 2). Por outro lado, $76,6 \%$ dos alunos que não freqüentaram tais cursinhos concorreram nos cursos do Grupo I, constituindo, assim, uma parcela menor. Em conseqüência, apesar da eficaz preparação dos alunos nos CPs, com 4,07 pontos a mais, em média, grande parcela dessas pessoas fica retida no "filtro", ocasionado pela intensa concorrência que ocorre nas RMs do Grupo I. A Tabela 6 apresenta as médias dos alunos não aprovados no processo seletivo. Nela, evidencia-se que grande número de alunos que freqüentaram CPs é reprovado, mesmo com notas médias superiores às que outros alunos necessitaram para o ingresso em especialidades menos concorridas.

\section{TABELA 6}

Número e notas médias dos candidatos que não foram aprovados no processo seletivo

\begin{tabular}{lcccc}
\hline & \multicolumn{2}{c}{ Freqüentaram CPs } & \multicolumn{2}{c}{ Não freqüentaram CPs } \\
\cline { 2 - 5 } & $\begin{array}{c}\text { Número de } \\
\text { candidatos não } \\
\text { aprovados }\end{array}$ & $\begin{array}{c}\text { Média } \\
\text { das notas }\end{array}$ & $\begin{array}{c}\text { Número de } \\
\text { candidatos não } \\
\text { aprovados }\end{array}$ & $\begin{array}{c}\text { Média } \\
\text { das notas }\end{array}$ \\
\hline Grupo I & 156 & 66,57 & 53 & 63,75 \\
Grupo II & 53 & 56,15 & 12 & 50,5 \\
Média & 209 & 63,68 & 65 & 60,08 \\
\hline
\end{tabular}

\section{CONCLUSÃO}

Constatou-se ser vantajosa a freqüência em Cursos Preparatórios para Residência Médica na preparação para o concurso de Residência Médica 2007 do HU-UFJF. Este processo demonstra como a desigualdade socioeconômica pode ser determinante na preparação de candidatos, na qual leva vantagem quem possui melhor condição financeira e pode financiar tais cursinhos. É de extrema importância, portanto, que órgãos e associações reguladores da educação médica brasileira estejam atentos.

É alarmante que o processo seletivo tradicional (modelo 90/10), realizado na instituição em questão e adotado quase absolutamente pelas instituições brasileiras que oferecem RMs, não corrobore adequadamente a formação acadêmica, o que contribui para o crescimento dos Cursos Preparatórios para Residência Médica, os quais têm fins lucrativos.

Em 2005, a Comissão Nacional de Residência Médica (CNRM) instituiu que seja opcional a cada instituição a adoção de um menor percentual de avaliação teórica (de 90\% para 50\%) e a possibilidade de utilizar essa diferença de $40 \%$ para avaliação prática. Trata-se de uma proposta adequada e passível de corrigir essa injustiça do modelo tradicional, além de incentivar o treinamento acadêmico nos hospitais universitários e nos estágios. Não se sabe o impacto que essas alterações teriam na procura por CPs para Residência Médica, já que estes cursos tendem a se adaptar, oferecendo treinamento para provas práticas.

É essencial realizar mais estudos em relação a essa questão. É evidente, entretanto, que o modelo de avaliação precisa 
ser revisto, discutido e modificado, na tentativa de valorizar a formação acadêmica. A solução para o processo seletivo para ingresso em RMs não é simples, mas já se constatou que a preocupação em relação ao tema tem mobilizado tanto as entidades responsáveis pelos programas de RM como órgãos ligados ao governo e a estudantes, os quais têm procurado discutir o assunto e propor alternativas, o que já é de suma importância.

\section{REFERÊNCIAS}

1. Medcel Residência Médica [homepage na Internet]. São Paulo: MedCel; [capturado em: 15 fev. 2007]. Institucional. Quem somos. Disponível em: <http://www.medcel.com. br/v2/quem-somos.php.>

2. Autenticmed [homepage na Internet]. Rio de Janeiro: Autenticmed; [capturado em: 15 fev. 2007]. Institucional. Quem somos. Disponível em: <http://www.autenticmed. com.br/institucional.asp>

3. Cursinhos se especializam em residência médica. J. P. on line [periódico na Internet]. $18 \mathrm{dez}$. 2006. [capturado em: 16 fev. 2007]. Disponível em: <http://www.jpjornal.com. br/news.php?news_id=40219>

4. Lopes AC. Residentes prontos para a vida real. Saúde Paulista [periódico na internet]. Jan. 2004 [capturado em: 18 fev. 2007]; 4(2). Disponível em: <http:/ / www.unifesp.br/ comunicacao/sp/ed12/opiniao.htm>

5. Chuen-Neto JA et al. Cursinhos Preparatórios Para Prova Residência Médica: Expectativas e Opiniões. In: Anais do 44 Congresso Brasileiro de Educação Médica; 2006 set. 2427; Gramado, Brasil.

6. Direção Executiva Nacional dos Estudantes de Medicina. Caderno de textos para subsidiar as discussões sobre Residência Médica nas coordenações locais da Denem [online]; Jul. 2005 [capturado em: 19 fev. 2007]. A prova prática e o Processo Seletivo de Residência Médica. Disponível em: <http://www.denem.org.br/2005/arquivos/ok1123095295737.doc>

7. Oficina Nacional de avaliação dos procedimentos da Prova Prática aplicada durante o Processo Seletivo de Residência Médica [homepage na internet]. Brasília: Comissão Nacional de Residência Médica. [capturado em: 20 fev. 2007]. Disponível em: <http:/ / portal.mec.gov.br/sesu/arquivos/pdf/cnrm/avalprovapratica.pdf>

8. Gomes FMS et al. $\mathrm{O}$ ensino médico na atenção primária em pediatria: um Programa para os Residentes no Centro de Saúde-Escola da FMUSP. Pediatria (São Paulo). 2001(1):52-59.

9. Gualberto LD. Residência Médica No Brasil. Revista Virtual de Medicina [periódico na internet]. Jan./mar. 1998 [capturado em: 22 fev. 2007]; 1(1): [aproximadamente 7 p.].
Disponível em: <http://www.medonline.com.br/med_ ed $/$ med $1 /$ residenc.htm $>$

10. Elias PE et al. Programas, Vagas e Perfil dos Residentes da Federação. Investigação sobre a necessidade de profissionais de saúde no país São Paulo: Universidade de São Paulo; 2005 .

11. Sistema da Comissão Nacional de Residência Médica (Instituições $x$ Programas $x$ Vagas) [Base de dados na internet]. Brasília: Ministério da Educação. [capturado em: 22 fev. 2007]. Disponível em: <http://www.mec.gov.br/sesu/ sist_cnrm/apps/Cons_Res_Inst.asp >

12. Residência Médica [homepage na internet]. Campinas: Hospital das Clínicas - UNICAMP; 2004. [capturado em: 22 fev. 2007]. Disponível em: <http://www.hc.unicamp. $\mathrm{br} /$ residencia/residencia.shtml $>$

13. Nunes MPT. A Residência Médica no Brasil: urgências e necessidades [periódico na internet]. 2006 [acesso: em 22 de Fevereiro de 2007]; 1(13). Disponível em: <http:// www.abem-educmed.org.br/publicacoes/boletim_virtual/volume_13/artigo_residencia.pdf.>

14. Revisão do Caderno de Avaliação pelo Comitê de Avaliação [homepage na internet]. Marília: Faculdade de Medicina de Marília; 21 fev. [capturado em: 24 fev. 2007]. Disponível em: <http://www.famema.br/avaliacao/manualavaliacao2006.pdf>

15. Cutait R, Correa, PAFP, Silva ED da et al. Resident selection for coloproctology: the Sirio Libanes Hospital experience. Rev bras. colo-proctol. oct./dec. 2006; 26(4): 394-398.

16. Martins MA. A Associação Brasileira de Educação Médica (ABEM) e a proposta de instituição de um Exame de Habilitação para o Exercício da Medicina no Brasil [homepage na internet]. Rio de Janeiro: ABEM; 22 set. 2005. [capturado em: 24 fev. 2007]. Disponível em: <http:/ /www.abemeducmed.org.br/pdf/posicao_exame_habilitacao.pdf>

ANEXO: O questionário está disponível utilizado está disponível para consulta em http://www.educacaomedica.org.br/ Anexos

\section{CONFLITOS DE INTERESSE}

Declarou não haver.

\section{ENDEREÇO PARA CORRESPONDÊNCIA}

Leonardo de Resende Sousa Oliveira

R. Delfim Moreira, 141 - apto. 602

Centro - Juiz de Fora

CEP.: 36010-570 / MG

E-mail: oileo@acessa.com 